\title{
Review of: "Effectiveness of CoronaVac in the setting of high SARS-CoV-2 P.1 variant transmission in Brazil: A test- negative case-control study"
}

Shrivardhan Dheeman

Potential competing interests: The author(s) declared that no potential competing interests exist.

I took a complete reading of the above-titled article. It is a nice study covering a new variant of SARS-CoV-2, called P.1 from Brazil. This study looks in the deepest of CoronaVac, a vaccine's effectiveness under high transmission rate. Based on method, and finding, briefly

0.50-fold reduction in SARS-CoV-2 infection under prescribed incubation, with a single dose, is interesting. Suggestion for the administration of at least one dose, as the conclusion is solely depending on the result and drawn well. 